\title{
Clinico-pathological changes in buffalo calves following oral exposure to Pasteurella multocida B:2.
}

\begin{abstract}
Background and aim: Pasteurella multocida B:2, which causes hemorrhagic septicemia of ruminants, is believed to enter the host via respiratory and oral routes. While the role of respiratory route of infection has been established, the present study describes the clinical and pathological alterations following oral exposure of buffalo calves to live wild-type Pasteurella multocida B:2. Methods: Nine 8-month-old buffalo calves were selected and divided into three groups. Calves of group 1 were exposed orally to $50 \mathrm{~mL}$ of the inoculums containing 109 colony-forming unit $(\mathrm{CFU}) / \mathrm{mL}$ of live Pasteurella multocida B:2. Calves of group 2 were exposed intra-tracheal to $5 \mathrm{~mL}$ of the same inoculums while calves of group 3 were exposed orally to $50 \mathrm{~mL}$ of phosphate-buffered saline. Results: Only one calf had to be euthanized at $48 \mathrm{~h}$ post-intra-tracheal exposure due to persistent clinical signs of hemorrhagic septicemia. The mean respiratory rate and rectal temperature had significantly increased in calves of groups 1 and 2. The pathological alterations included submandibular and brisket edema, generalized lymphadenopathy, acute fibrinous pneumonia, acute colitis and hemorrhagic typhilitis and proctitis. Nevertheless, oral infection with $109 \mathrm{CFU} / \mathrm{mL}$ of live wild-type P. multocida B:2 failed to produce a typical clinical disease. However, P. multocida $\mathrm{B}: 2$ was present along the gastro-intestinal tract, including the rectum of the calf that succumbed to the infection at $48 \mathrm{~h}$ post-intra-tracheal exposure. Conclusions: The presence of P. multocida B:2 along the gastro-intestinal tract is of concern since transmission via the gastro-intestinal tract of diseased animals is strongly possible.
\end{abstract}

Keyword: Clinical signs; Pathology; Pasteurella multocida B:2; Oral exposure; Buffalo. 